\title{
New approaches in the treatment of HIVIAIDS - focus on maraviroc and other CCR5 antagonists
}

\author{
Hans P Schlecht ${ }^{1}$ \\ Sarah Schellhorn ${ }^{2}$ \\ Bruce J Dezube ${ }^{3}$ \\ Jeffrey M Jacobson' \\ 'Department of Medicine (Infectious \\ Diseases), Hahnemann University \\ Hospital, Drexel University College \\ of Medicine, Philadelphia, PA, \\ USA; ${ }^{2}$ Department of Medicine, \\ ${ }^{3}$ Department of Medicine \\ (Hematology/Oncology), Beth Israel \\ Deaconess Medical Center, Harvard \\ Medical School, Boston, MA
}

\begin{abstract}
Treatment of HIV-1 infection has produced dramatic success for many patients. Nevertheless, viral resistance continues to limit the efficacy of currently available agents in many patients. The CCR5 antagonists are a new class of antiretroviral agents that target a necessary coreceptor for viral entry of many strains of HIV-1. Recently, the first agent within this class, maraviroc, was approved by a number of regulatory agencies, including the Food and Drug Administration. Herein we review the role of the CCR5 receptor in HIV-1 infection and potential methods to target it in anti-HIV-1 therapy. We review the various categories of agents and discuss specific agents that have progressed to clinical study. We discuss in detail the recently approved, first in class CCR5 antagonist, maraviroc, and discuss aspects of resistance to CCR5 antagonism and the potential role of CCR5 antagonism in the management of HIV-1 infection.
\end{abstract}

Keywords: CCR5, HIV-1 tropism, coreceptor, maraviroc, viral entry, chemokine receptor

\section{Introduction}

The widespread use of highly active antiretroviral therapy (HAART) has profoundly affected the treatment and epidemiology of HIV-1 infection within the developed world, where HIV-1 infection has largely become a chronic illness (Palella et al 1998). HAART's dramatic success has contributed in saving at least 3 million years of life in the US since 1989 and will aid a patient living with HIV-1 to live more than 13 years longer today than if they had been diagnosed in 1988 (Walensky et al 2006). Despite tremendous impact, patient benefit from HAART is not simple or uniform; $25 \%$ of patients starting HAART either do not achieve viral suppression or lose it within 2 to 3 years, frequently due to viral resistance (Bartlett et al 2001; Mocroft et al 2002; Holmberg et al 2003). Moreover, the rate of acquired resistance in patients recently infected with HIV-1 has grown from 13.2\% during the period 1995 to 1998 to $24.1 \%$ during 2003 to 2004, including some with rapid progression to AIDS (Markowitz et al 2005; Shet et al 2006).

In spite of the recalcitrant nature of HIV-1 viral resistance, recent advances have been substantial. The approval of darunavir, a protease inhibitor with potent efficacy against multi-drug resistant strains of HIV-1, has helped shift the emphasis of HIV-1 salvage treatment from preserving immune function to complete suppression of viral replication and immune reconstitution (Hammer et al 2006). With the advent of additional classes of agents such as CCR5 and integrase antagonists, patients with extensive multi-drug resistance will likely be able to achieve an undetectable viral load. Nevertheless, resistance to these new agents has already been seen in vitro and will inevitably arise clinically (Mosley et al 2006; Cooper et al 2007; Steigbigel et al 2007). HIV clinical investigators must be prepared to overcome these defenses by refining the current use of HAART and wisely integrating new agents, such as CCR5 antagonists, in order to minimize the impact of HIV-1 viral resistance on patients and populations (Schooley and Mellors 2007). 
Herein we review the role of the CCR5 receptor in HIV-1 infection and potential methods to target it in antiHIV-1 therapy. We review the various categories of agents and discuss specific agents that have progressed to clinical study. In particular, we discuss in detail the recently approved, first in class CCR5 antagonist, maraviroc, and discuss aspects of resistance to CCR5 antagonism and the potential role of CCR5 antagonism in the management of HIV-1 infection.

\section{Viral entry and CCR5}

The mechanism by which HIV-1 infects cells was a prime target for early HIV-1 researchers. In 1984, the CD4 molecule was identified as necessary for HIV-1 replication within host cells (Dalgleish et al 1984). Later studies showed that CD4 alone was not sufficient for HIV-1 infection of a host cell and 2 years later, the chemokine receptor CCR5 was identified as a major co-receptor (Dragic et al 1996).

The proteins required for HIV-1 entry into the cell are encoded by the env gene, the product of which is the precursor to both the gp120 and gp41 glycoproteins (Chan et al 1997).
Gp120 associates with the CD4 receptor on the surface of the host cell; gp41 spans the viral envelope and mediates viral fusion with the host cell. The two glycoproteins associate non-covalently on the viral envelope as a heterodimer and then further assemble as a trimer to form the fusion mediating structure (Kwong et al 1998).

On exposure of the virus to a cell expressing CD4, gp120 interacts with the CD4 molecule, thereby inducing a conformational change in gp120 that enables binding to the chemokine receptor (see Figure 1). Binding of gp120 to the chemokine receptor (either CCR5 or CXCR4) generates a conformational change in gp41, leading to insertion of a lipophilic region of gp41, known as the fusion peptide, into the lipid bilayer of the host cell. A transitional intermediate state is created in which gp41 is inserted into both the viral envelope and the cellular membrane. The virus and the cell are brought together as gp41 folds on itself in a hairpin structure, thereby bringing the viral envelope into close proximity with the cell membrane of the CD4+ host cell. Fusion is initiated, and the viral core contents are spilled into the cytoplasm (Chan et al 1998; Eckert et al 2001).

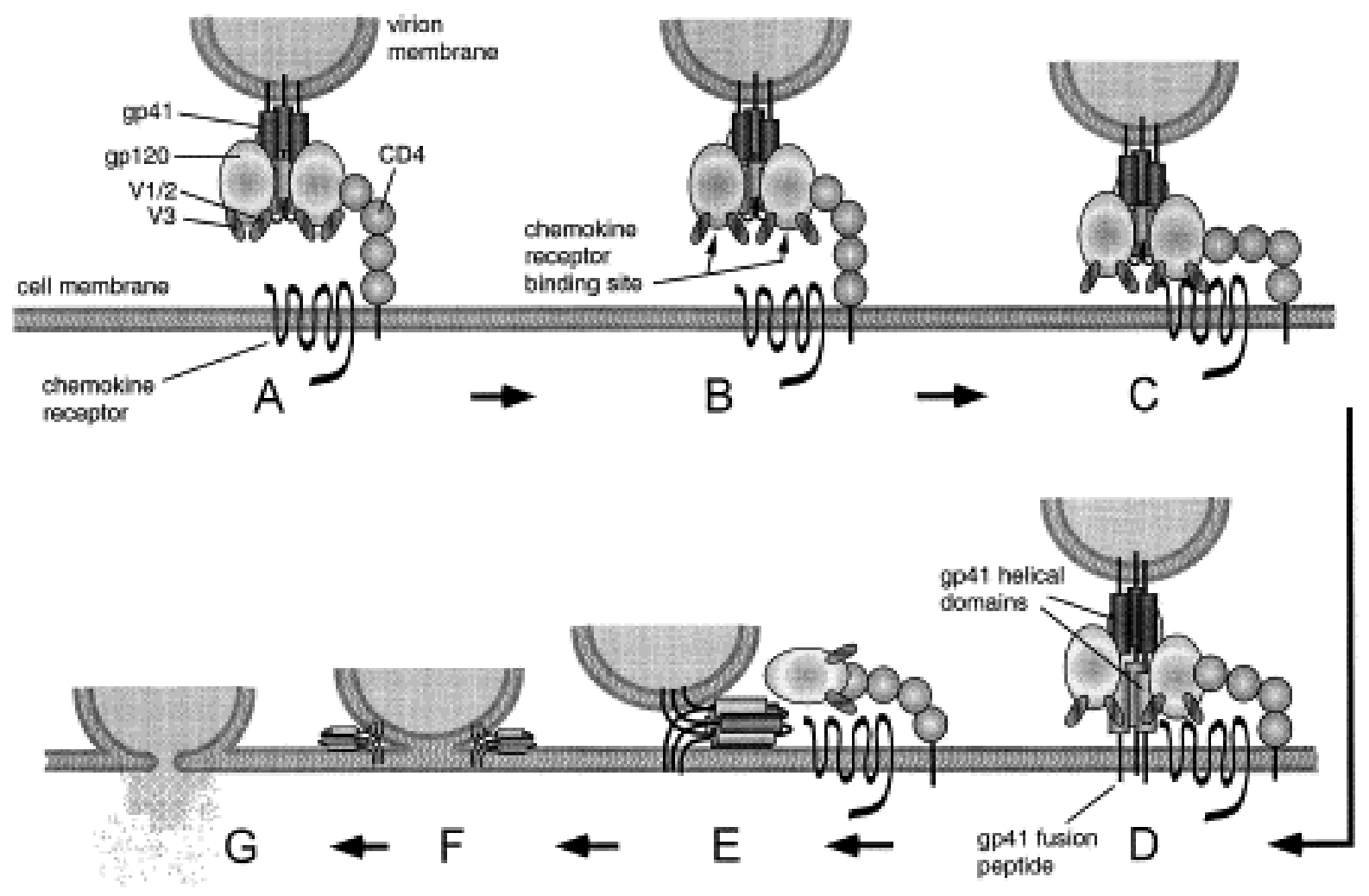

Figure I HIV-I entry via CD4 and coreceptor binding gP I 20 binds to CD4 (A) and undergoes conformational changes that expose the co-receptor binding site (B) and enable binding to the chemokine receptor (C). Structural changes are then induced in gP4I that extend the helical domains to form a 'pre-hairpin intermediate' (D). The hydrophobic fusion peptide inserts into the target cell membrane, causing gP4I to span between the virus and cell membranes. The gP4I helices then fold into a six-helix bundle, bringing together the $\mathrm{N}$-terminal and $\mathrm{C}$-terminal domains and thus the viral and cellular membranes (E). Contact between the membranes allows mixing of the outer leaflets followed by the development of a fusion pore (G). gp I20 is omitted from panels F and G for the sake of clarity. Reprinted with permission from Starr-Spires LD, Collman RG. 2002. HIV-I entry and entry inhibitors as therapeutic agents. Clin Lab Med, 22:68I-70I. Copright 2002 @ Elsevier. 

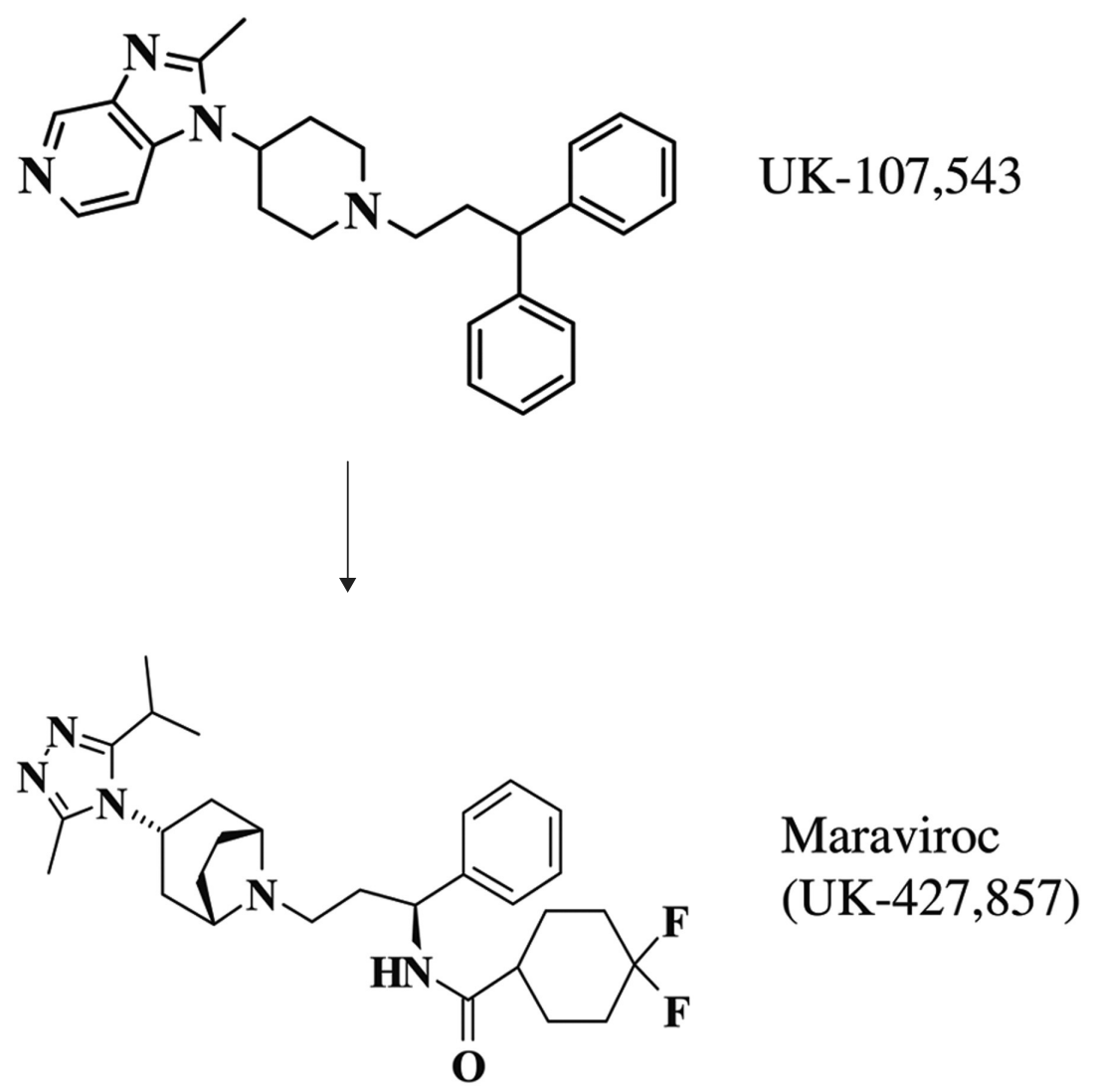

Figure 2 Development of maraviroc from the high-throughput screening hit UK-107,543. Reprinted with permission from Dorr P,Westby M, Dobbs S, et al 2005. Maraviroc (UK-427,857), a potent, orally bioavailable, and selective small-molecule inhibitor of chemokine receptor CCR5 with broad-spectrum anti-human immunodeficiency virus type I activity. Antimicrob Agents Chemother, 49:4721-32. Copyright ( American Society of Microbiology.

\section{Structure, location, and function of CCR5}

Similar to other G-protein coupled receptors, chemokine receptors have a seven transmembrane structure. The N-terminus is extracellular and participates in binding of the chemokine; the $\mathrm{C}$-terminus is intracellular and serves as the site for $\beta$-arrestin binding. $\beta$-arrestins facilitate G-protein independent cell signaling in addition to binding the chemokine receptor to clathrin for endocytosis and subsequent recycling (Zhao et al 1998; Oppermann 2004; Signoret et al 2005). Two separate co-receptors for HIV-1 infection have been identified: CCR 5 and CXCR4. There is substantial genetic variability within the amino acid sequence of gp120 molecule on the viral envelope, determining to which chemokine receptor the gp120 will bind (Pastore et al 2006).

The ligands for CCR5 and CXCR4 are chemokines, which are small molecules in the cytokine family that promote chemotaxis and cellular activation; on binding to their receptor on the target cell, an intracellular signaling cascade is initiated via a G-protein release from the intracellular domain of the receptor (Oppermann 2004). Native ligands for CCR5 include chemokines MIP-1 $\alpha$ (macrophage inflammatory protein), MIP-1 $\beta$, and RANTES (regulated on activation, normal T-cell expressed and secreted), which have been giving standard nomenclature CCL3, CCL4, and CCL5, respectively; CCR5 is expressed on immune cells, including effector and memory T-cells, natural killer cells, and antigen presenting cells (Blanpain et al 1999; Ruffing et al 1998). As is exemplified by CCR 5 and its ligands, the host defense mechanism is both complex and redundant. A single chemokine receptor can be activated by different chemokines, and similarly, a single chemokine may also activate multiple different chemokine receptors. This redundancy provides a back up plan to counteract the strategies and mutations microbes use to circumvent the host defense system.

CCR5 is heavily involved in both initiating and amplifying the immune response. At the time of microbial invasion, conserved antigens on the microbe bind to toll-like receptors on the surface of macrophages and immature dendritic cells, which release cytokines and chemokines in response. CCR5 expressing cells are then drawn toward the site of invasion by the high concentration of CCR5 binding chemokines; they ingest 
pathogens, mature, and then migrate to lymphoid tissue where they then prime the adaptive immune response by presenting the microbial antigens to naive B- and T-lymphocytes (Wong et al 2003). Antigen-specific T-lymphocytes can also release CCR5-binding chemokines, which bind to endothelial cells and components of the extracellular matrix. CCR5 expressing cells roll along the surface of the endothelium and are drawn to the increased chemokine gradient at the site of infection (Wong et al 2003, Luther et al 2001). Of note, while CCR5 has several identified agonists, CXCR4 has only one (Stromal derived factor-1).

\section{Distribution of CCR5-tropic virus}

\section{in the HIV-positive}

HIV-1 is capable of using either CCR5 or CXCR4 as a co-receptor in vivo; in vitro, it has been shown to use other chemokine receptors (Unutmaz et al 1998). Some strains of HIV-1, known as R5, use only CCR5 as the co-receptor to gain access to the host cell. Other strains, X4 strains, use only CXCR4 as its co-receptor. Dual or mixed (D/M) strains, also known as R54 strains, can use both CCR5 and CXCR4.

In early infection, the vast majority $(70 \%-80 \%)$ of strains isolated from treatment-naïve patients are CCR5-tropic or R5 viruses (Brumme et al 2005; Moyle et al 2005), whereas X4 strains, previously known as syncytium-inducing variants, are seen in $30 \%-60 \%$ of treatment-experienced patients and arise later in the natural history of HIV-1 infection. They are associated with accelerated progression to AIDS and lymphocyte depletion. It is unclear whether the appearance of variants that use CXCR4 as a co-receptor is a cause or an effect of immunodeficiency (Koot et al 1993; Connor et al 1997; Melby et al 2006; Wilkin et al 2007). It should be noted that R5 strains still persist late into the course of disease, so the CCR5 co-receptor may still be an important pharmaceutical target even in advanced stages of disease (Wilkin et al 2007).

\section{Natural variant of CCR5}

In 1996, a 32 base pair mutation in the gene for CCR5, known as $\Delta 32$, was shown to protect against HIV-1 infection (Huang et al 1996). The mutant allele codes for a dysfunctional truncated protein that is not expressed on the surface of the cell. The frequency of the allele is approximately $10 \%-14 \%$ in the white population, particularly those of Northern European descent, but is seldom seen in Asians or Africans; approximately $1 \%$ of the Caucasian population is homozygous for the deletion (Liu et al 1996). Infection of $\Delta 32$ homozygotes is exceedingly rare, and when it occurs is caused by viruses using CXCR4 as a co-receptor for viral entry; heterozygotes can be infected with the HIV-1 virus but tend to have a longer disease course (Dean et al 1996; Liu et al 1996; Samson et al 1996). Patients who are homozygous for the $\Delta 32$ mutation are phenotypically normal, without readily apparent negative effects or decreased life expectancy. The preserved functional phenotype of $\Delta 32$ homozygotes is attributable to the redundancy present in the host defense system. Interestingly, mutations in murine CXCR4 (or its only known native ligand, Stromal factor-1) are lethal in the embryo (Lapidot 2001).

However, recent evidence suggests $\Delta 32$ homozygotes may have immunological consequences. Early researchers proposed that the mutation, in its early age, may have provided protection against Yersinia pestis (Stephens et al 1998), the microbial agent of the bubonic plague; others have suggested that protection against smallpox may have been the survival advantage (Galvani et al 2003). The area remains controversial, and recent population studies indicate that evolution of CCR5 may have been neutral (Sabeti et al 2005). Another study demonstrated longer survival and delayed rejection of renal allografts in $\Delta 32$ homozygotes (Fischereder et al 2001), and CCR5 32 may be protective against the development of rheumatoid arthritis and persistent hepatitis B infection (Prahalad et al 2006; Thio et al 2007). The $\Delta 32$ mutation has also been associated with increased mortality from encephalopathy caused by West Nile Virus (Glass et al 2006). Additional studies will likely reveal other previously unrecognized complications or benefits associated with absence, dysfunction, or blockade of CCR5.

\section{Targeting CCR5}

A number of potential mechanisms are under investigation to inhibit HIV-1 binding and fusion to human cells. These include agents to block CD4 binding by viral gp120, inhibit CCR5 or CXCR4 co-receptor binding by gp120, as well as inhibit gp41 mediated fusion of the viral and cellular lipid bilayers as the Food and Drug Administration (FDA)approved agent enfuvirtide does (Guo et al 2003; Jacobson et al 2004; Oldfield et al 2005; Kadow et al 2006; Moyle et al 2007). While it is beyond the scope of this review to discuss every agent, we will review developmentally advanced agents and the various tactics under study for the antagonism of CCR5.

With the initial discovery that CCR5 was an HIV-1 coreceptor and its endogenous ligands, (MIP-1 $\alpha$, MIP-1 $\beta$, and RANTES) able to suppress HIV-1 replication, efforts centered on pharmacologically reproducing the effects of 
these chemokines. This antiviral effect is related to the ligands' ability to internalize the receptor and deprive it from being expressed on the cell surface (Cocchi et al 1995). The promise of these modified chemokines and other novel agents has been difficult to bring to fruition thus far and their future remains uncertain (Simmons et al 1997; Qin et al 2003; Hartley et al 2004; Anderson and Akkina 2007). Another unique approach attempts to employ a zinc finger nuclease to bind, cleave, and cause mutagenesis in the CCR5 gene and thereby inhibit normal transcription and protein expression (Jouvenot et al 2005; Mani et al 2005).

Monoclonal antibodies targeting CCR5 have also been developed with CCR5mAb004 of Human Genome Sciences (Rockville, MD, USA), and PRO 140 of Progenics Pharmaceuticals (Tarrytown, NY, USA) progressing to early phase human trials. Each is a mouse-derived but humanized monoclonal antibody capable of blocking CCR5 without activation of the receptor (Olson et al 1999; Roschke et al 2004). A phase I study in 63 HIV-positive patients with CCR5-tropic virus demonstrated CCR5mAb004 to be safe and well tolerated with substantial anti-viral efficacy for up to 28 days after a single dose (Lalezari et al 2006). Subjects were randomized to 5 cohorts with an 8:2 ratio of placebo to active drug. Subjects were infused with a single dose of placebo or CCR5mAb004 at 1 of 5 dosages, $0.4,2,8,20$, or $40 \mathrm{mg} / \mathrm{kg}$ of body weight, and followed for 8 weeks. Greater antiviral response was observed at higher doses with $55 \%$ of subjects receiving doses of $8 \mathrm{mg} / \mathrm{kg}$ or greater and $50 \%$ in the highest dose cohort demonstrating at least a $1 \log _{10}$ drop in HIV viral load. Further investigation of a more potent, next generation antibody is anticipated.

PRO 140, considered a fast-track product by the FDA, recently reported positive proof-of-concept study results. A phase I study in 39 HIV-positive patients with CCR5-tropic virus demonstrated PRO 140 to be safe, well tolerated and a potent inhibitor of HIV-1 replication with a mean maximal viral load reduction of $1.83 \log _{10}$ in the highest dose group ( $5 \mathrm{mg} / \mathrm{kg}$ ) after a single dose (Jacobson et al 2007). All patients receiving the highest dose had a viral load reduction of at least $1 \log _{10^{*}}$. PRO140 binds to the $\mathrm{N}$ terminus and second extracellular domain of CCR5 and acts as a direct competitive inhibitor of HIV binding. The small-molecule antagonists to be discussed bind in a hydrophobic pocket of CCR5 formed by transmembrane helices, and act through allosteric effects. As such, the two classes of CCR5 antagonists work through different mechanisms, and virus resistant to the small molecule antagonists maintain sensitivity to PRO140 (Kuhmann et al 2004; Marozsan et al 2005). In addition, in vitro studies demonstrate potentially synergistic effects with the small-molecule CCR5 antagonists (Murga et al 2006; Ji et al 2007). Though PRO 140 requires parenteral administration, the interval of administration ultimately determined could be long enough to be reasonably convenient. The preliminary results are encouraging.

By far the largest group and furthest in development of CCR5 antagonists are the small-molecule inhibitors. Typically cheaper to produce than peptides with better oral bioavailability, these agents also demonstrate significant potency across diverse clades with half maximal inhibitory concentrations $\left(\mathrm{IC}_{50}\right)$ measured in nanomoles. As is the case with many small-molecule inhibitors of G-coupled proteins, these agents appear to bind a pocket within the transmembrane helices, alter extracellular CCR5 conformation, and thereby inhibit HIV-1 binding (Dragic et al 2000). Agents that have progressed from simple labels to scientific names end in the suffix-viroc, eg, vicriviroc, to denote their action of viral receptor occupancy. Small-molecule antagonists include: TAK-779, TAK-220, Tak-652, AK602/ ONO4128/GSK-873140/aplaviroc, SCH-C/SCH-351125, SCH-D/SCH-417690/vicriviroc, and UK-427,857/maraviroc. In addition to the recently approved maraviroc, which we discuss in greater detail in the following section, a number of other agents merit discussion.

The first small-molecule CCR5 antagonist published was TAK-779 of Takeda Chemical Industries (Osaka, Japan) (Baba et al 1999). Though a potent inhibitor of CCR5-tropic HIV-1 in vitro, development was curtailed due to the need for subcutaneous administration and the attendant difficulties with injection site reactions. More recent focus has been placed on TAK-220 and TAK-652, each with nanomolar inhibition of HIV-1 in vitro and oral bioavailability (Baba et al 2005; Takashima et al 2005). A single-dose phase I study has been completed for TAK-652 and demonstrates favorable initial safety and pharmacokinetic data (Baba et al 2005). Further clinical data are awaited.

Spirodiketopiperazine derivatives have been under active development by Ono Pharmaceuticals (Osaka, Japan) in collaboration with GlaxoSmithKline (Research Triangle Park, NC, USA). E913 potently suppresses the replication of laboratory and primary CCR5-tropic HIV-1 strains, including viruses resistant to available antiretroviral medications (Maeda et al 2001). However aplaviroc (AK602/ONO4128/GSK873140) demonstrates a 2-fold increase in activity versus HIV-1 isolates at sub-nanomolar concentration both in vitro and in vivo mouse model, and became the lead analogue under development (Maeda et al 2004; Nakata et al 2005). In early phase study, a maximum dose of $600 \mathrm{mg}$ twice a day for 
10 days demonstrated a $1.66 \log _{10}$ mean reduction in viral load and was well tolerated (Lalezari et al 2005). However during phase IIb trials three subjects developed severe hepatotoxicity, which was deemed secondary to drug-induced hepatitis. Moreover, 1 of the first 44 subjects enrolled in a phase III trial of aplaviroc developed severe hepatotoxicity, and the trials and development of aplaviroc were stopped (Crabb 2006).

CCR5 antagonist, SCH351125 or SCH-C, was the first agent developed by Schering-Plough Corporation (Kenilworth, NJ, USA) due to its antiviral potency-- nanomolar $\mathrm{IC}_{50}$ concentrations for a range of CCR5-tropic HIV-1 isolates-- and advantageous pharmacokinetic characteristics (Strizki et al 2001). The first CCR5 antagonist to enter clinical efficacy studies, SCH-C at a dose of $25 \mathrm{mg}$ twice daily demonstrated a $0.5-1 \log$ drop in viral load in 10 of 12 subjects (Reynes et al 2002). However, antiviral effect was not uniform and QTc interval prolongation prompted further optimization work. This resulted in Sch-417690 or SCH-D, later known as vicriviroc. Vicriviroc is 2-40 times more potent than SCH-C against primary HIV-1 isolates in vitro, has excellent oral bioavailability, and an improved safety profile (Strizki et al 2005).

In early phase study, vicriviroc was well tolerated up to $50 \mathrm{mg}$ twice daily and produced 1.5 to $1.6 \mathrm{log}$ reductions in HIV-1 viral load at doses of $25 \mathrm{mg}$ and $50 \mathrm{mg}$ twice daily in treatment-naïve subjects (Schurmann et al 2007). However, treatment-naïve subjects with R5-tropic virus fared less well in a phase II study comparing vicriviroc to efavirenz (EFV), both combined with zidovudine/lamivudine (AZT/3TC as fixed dose Combivir ${ }^{\circledR}$ [GlaxoSmithKline]). Subjects began the study receiving either placebo or vicriviroc monotherapy $(25,50$, or $75 \mathrm{mg}$ once daily) for 2 weeks. Thereafter AZT/3TC was added to subjects receiving vicriviroc, while subjects receiving placebo were given EFV and AZT/3TC instead. Though vicriviroc produced a $0.9-1.3 \log _{10}$ greater reduction in viral load than placebo, preliminary analysis demonstrated virologic breakthrough (RNA $\geq 50$ copies $/ \mathrm{mL}$ ) during follow-up in 2 of 24 (8\%) of the placebo/AZT/3TC/ EFV group versus 13 of 23 (57\%), 10 of 22 (45\%), and 5 of $23(22 \%)$ in the 25,50 , and $75 \mathrm{mg}$ arms of vicriviroc, respectively (Greaves et al 2006). The study was subsequently terminated prematurely on recommendation of the data safety monitoring board. Neither tropism shifts nor elevated vicriviroc $\mathrm{IC}_{50}$ measurements accounted for the higher rate of virologic breakthrough. However, all subjects with virologic breakthrough and genotypic data available had treatment-emergent M184V mutations, suggesting that vicriviroc was underdosed. Such results have provoked calls that there is a near unassailable standard of care in treatment-naïve regimens, eg, EFV-based, and that these potent regimens should be embraced (Deeks 2006).

Phase II vicriviroc results have been more encouraging among treatment-experienced subjects. In an AIDS Clinical Trial Group study, ACTG A5211, 24 week and recently presented 48 week data has shown that vicriviroc provided a significant advantage over placebo when added to optimized background regimen (Gulick et al 2007a, b). Both the $10 \mathrm{mg}$ and the $15 \mathrm{mg}$ once daily doses were shown to provide durable virologic suppression and significant immunologic benefit: HIV-1 RNA decreases of 1.9 and $1.4 \operatorname{logs}$, and CD4+ cell count increases of 96 and 130 cells/ $\mathrm{mm}^{3}$ were seen in the $10 \mathrm{mg}$ and $15 \mathrm{mg}$ arms, respectively. Moreover, $37 \%$ of subjects in the $10 \mathrm{mg}$ vicriviroc group and $27 \%$ of those in the $15 \mathrm{mg}$ group had a HIV-1 viral load below 50 copies/mL at week 48 . However, the $5 \mathrm{mg}$ dose study arm was discontinued early based on recommendation from the Study Monitoring Committee and the study was also unblinded following reports of 5 malignancies in subjects receiving vicriviroc. Further follow-up of 39 subjects enrolled in an open label extension study reported no new malignancies and in September 2007, it was announced that two phase III studies, entitled VICTOR-E3 and VICTOR-E4, had begun to evaluate the safety and effectiveness of $30 \mathrm{mg}$ of vicriviroc plus optimized background therapy (OBT) in treatment-experienced subjects (Gulick et al 2007c).

\section{Maraviroc}

With greater understanding of the role and impact of the CCR5 receptor in the transmission and progression of HIV-1 disease, numerous efforts to pharmacologically target the CCR5 receptor began. Drug development work at Pfizer Inc. (Kent, UK) resulted in the development of UK-427,857 or maraviroc (Dorr et al 2005; Wood and Armour 2005). Opening effort centered on a high throughput screening assay employing HEK-293 cells to express CCR5 and used candidate drug antagonism of radiolabelled MIP-1 $\beta$ binding as the basis of the screen resulting in hits UK-107,543 and UK-179,645.

Following intensive structure-activity relationship (SAR) studies and numerous structural modifications via modern medicinal chemistry, UK-427,587, or 4,4' -difluoro-N-[(1S)-3 - [(3 - exo)-3 - [3 - methyl-5 (1-methylethyl)-4H-1,2,4-triazol-4-yl]-8-azabicyclo[3.2.1] oct-8-yl]-1-phenylpropyl]cyclohexanecarboxamid, became the lead analogue (see Figure 1). With its ability to block CCR5 binding and signaling at nanomalor concentrations plus no 
problematic cytochrome P450 D2 interaction or cardiac ion channel binding, and favorable permeability characteristics, further investigation began of this potent inhibitor of laboratory optimized HIV-1 isolates (mean inhibitory concentration of $90 \%$ of isolates or $\mathrm{IC}_{90}$ of $2.0 \mathrm{nM}$ ).

Additional work demonstrated encouraging pharmacokinetic data, and high selectivity for the CCR5 receptor compared to other pertinent receptors, eg similar chemokine receptors. The antiretroviral activity of UK-427,587 proved potent against primary HIV-1 isolates from a number of clades, as well as drug-sensitive and drug-resistance clinical isolates of HIV-1 both as monotherapy and in concert with additional antiretrovirals. UK-427,587's mechanism of action was also confirmed to be strictly attributable to CCR5 blockade and no other antiviral means, eg, cell lysis.

Measurements made in first-in-man studies closely matched expectations derived preclinically (Dorr et al 2005; Wood and Armour 2005). Single doses were tolerable up to $900 \mathrm{mg}$, multiple doses to $600 \mathrm{mg}$ daily, and twice daily doses of $100 \mathrm{mg}$ of maraviroc continuously maintained plasma exposure above the $\mathrm{IC}_{90}$. No worrisome laboratory or electrocardiographic findings were observed and phase IIa efforts with 10 days of maraviroc monotherapy in the HIV-1 infected commenced shortly thereafter.

These two studies (A4001007 and A4001015) were combined for analysis and reported in November 2005 (Fätkenheuer et al 2005). Investigators randomized 82 CCR5-positive subjects to receive 10 days of treatment with maraviroc (25 mg, $100 \mathrm{mg}$ or $300 \mathrm{mg}$ once daily or $50 \mathrm{mg}$, $100 \mathrm{mg}, 150 \mathrm{mg}$ [fed and fasted] or $300 \mathrm{mg}$ twice daily) or placebo. Baseline subject characteristics were similar across treatment groups and barring one subject inadvertently enrolled with $\mathrm{D} / \mathrm{M}$ infection, subjects receiving $100 \mathrm{mg}$ twice daily or more demonstrated robust HIV-1 suppression. Maximum viral load reductions averaged -1.68 (range -2.10 to -1.37 ) for the $100 \mathrm{mg}$ twice daily group and peaked at -1.84 (range -2.42 to -1.49 ) for $300 \mathrm{mg}$ twice daily. These values frequently occurred after day 11 and likely reflect the prolonged occupancy of the CCR5 receptor by maraviroc, a phenomenon also reported for other CCR5 antagonists (Lalezari et al 2005; Schurman et al 2007). Three subjects were excluded from analysis; the aforementioned subject inadvertently enrolled with $\mathrm{D} / \mathrm{M}$ infection, a subject who withdrew consent, and a subject who discontinued treatment prior to day 3 secondary to a moderate headache felt to be unrelated to their $100 \mathrm{mg}$ once daily dose. Maraviroc was generally well tolerated, except for one case of severe diarrhea in the $150 \mathrm{mg}$ twice daily (fed) group which started on day 1 and resolved after 11 days without intervention. The most common mild or moderate side effects were headache, asthenia, dizziness, gingivitis, and nausea. Overall these results were one of the first to demonstrate the viability of targeting the CCR5 receptor in the treatment of HIV-1 infection.

Late phase efforts in maraviroc's development have targeted both treatment-naïve and treatment-experienced patients. Among treatment-naïve patients, maraviroc is being investigated in the MERIT study. This ongoing phase IIb/III study had interim 48-week data presented at the 4th IAS Conference on HIV Pathogenesis, Treatment and Prevention held July, 2007 (Saag et al 2007). This study tests the hypothesis that maraviroc is not inferior to EFV when used in combination with AZT/3TC (provided as fixed dose Combivir) in CCR5-tropic patients without resistance to AZT, 3TC or EFV. Subjects were stratified at entry by HIV-1 viral load ( $>$ or $<100,000$ copies/mL) and also geographic location (Northern vs Southern Hemisphere). Of note, in an effort to minimize discontinuance due to medication intolerance, especially AZT, subjects who experienced toxicity were allowed to substitute an alternative nucleoside reverse transcriptase inhibitor. The primary endpoint was the proportion of patients who achieved a viral load $<50$ copies/mL through 48 weeks, which proved important to the investigators' conclusions.

Initially planned with three treatment groups, maraviroc $300 \mathrm{mg}$ once or twice daily or EFV, the data safety and monitoring board closed the once-daily maraviroc arm early because of inadequate response rates. The remaining study arms recruited 721 subjects with a mean age of 37 years, $79 \%$ men $/ 21 \%$ women, roughly $50 \%$ white $/ 35 \%$ black, and roughly 400 from the Northern Hemisphere vs 300 from the Southern Hemisphere. At baseline, subject characteristics were similar between the EFV and maraviroc groups: median CD4 counts of 241 and 254 cells $/ \mathrm{mm}^{3}$, respectively, and mean viral load of roughly $4.9 \log$ copies $/ \mathrm{mL}$ each.

By 48 weeks, $65.3 \%$ of the maraviroc arm and $69.3 \%$ of the EFV arm achieved a viral load $<50$ copies $/ \mathrm{mL}$. This margin of difference failed to meet the preselected criteria for noninferiority as the lower bound of the $97.5 \%$ confidence interval was less than $-10 \%$ (observed noninferiority margin $-10.9 \%$ ). Using an endpoint of $<400$ copies $/ \mathrm{mL}$ at 48 weeks, twice daily maraviroc met noninferiority criteria as $70.6 \%$ of subjects in the maraviroc arm and $73.1 \%$ of the EFV arm achieved the endpoint. Also by 48 weeks, a greater increase in mean CD4 cell count was observed in the maraviroc treated subjects than in the EFV treated $\left(170\right.$ cells $/ \mathrm{mm}^{3}$ vs 144 cells $\left./ \mathrm{mm}^{3}\right)$ though this difference likely has little clinical relevance. 
Two subgroup analyses are of particular note. Among subjects with baseline viral loads $<100,000$ copies $/ \mathrm{mL}$ EFV and maraviroc had similar rates of suppression to $<50$ copies $/ \mathrm{mL}$ ( $71.6 \%$ vs $69.6 \%$ respectively). However, EFV was superior to maraviroc when baseline viral load was $>100,000$ copies (proportion with viral loads $<50$ copies $/ \mathrm{mL}, 66.6 \%$ vs $59.6 \%$ ). The two geographic regions also demonstrated a difference in response. Higher rates of response were observed with EFV in subjects from the Southern hemisphere (proportion with viral loads $<50$ copies $/ \mathrm{mL}, 71.0 \%$ vs $62.1 \%$ ) while similar rates of response were observed in subjects from the Northern Hemisphere. No underlying mechanism(s) has been clearly identified for this difference though clade variation between hemispheres may play a role.

Rates of study drug discontinuation were similar in the two groups (26.9\% for maraviroc and $25.2 \%$ for EFV) though reasons did differ. Subjects receiving EFV were more likely to stop due to adverse events ( $13.6 \%$ vs $4.2 \%$; typically neuropsychiatric side effects such as abnormal dreams and dizziness) while subjects receiving maraviroc experienced higher rates of virologic failure (11.9\% vs $4.2 \%$ in the EFV arm). Subjects taking maraviroc had fairly more frequent cases of bronchitis, and nose and throat infections.

Further safety analyses demonstrated similar proportions of severe adverse events as well as severe (grade 3 or 4) liver enzyme disturbances, which were infrequent in both groups but nevertheless helped prompt an FDA "black box" warning regarding potential hepatotoxicity. Rates of new malignancies also did not differ between the treatment groups, which is a reassuring finding after initial concerns regard increased risk of malignancy with vicriviroc and potentially all CCR 5 antagonists. Overall, maraviroc performed very well in the MERIT study but fell just short of rivaling EFV, an agent considered by some to be a standard worth embracing (Deeks 2006).

Two identical studies, MOTIVATE 1 and MOTIVATE 2, are investigating maraviroc in treatment-experienced
CCR5-tropic HIV-1 positive patients (Lalezari et al 2007; Nelson et al 2007). Identical in design, these phase IIb/III studies included subjects with: resistance to and/or $\geq 6$ months experience with at least one antiretroviral agent from each of the three approved oral drug classes (at least 2 agents for protease inhibitors), HIV-1 viral load of $\geq 5,000$ copies $/ \mathrm{mL}$ and either off treatment or on a stable but failing antiretroviral regimen for at least four weeks. Subjects were stratified by enfurvitide use as well as viral load $>$ or $<100,000$ copies $/ \mathrm{mL}$. Applying genotypic and phenotypic test results, subjects were randomized to receive OBT plus placebo, maraviroc once daily or maraviroc twice daily (subjects receiving a PI other than tipranavir and/or delavirdine in their OBT received $150 \mathrm{mg}$ while remaining subjects received $300 \mathrm{mg}$ ) in a 1:2:2 ratio.

Interim results from planned 24-week analyses of both trials were very similar (see Table 1). MOTIVATE 1 enrolled 601 subjects mainly from North America, while MOTIVATE 2 enrolled 475 individuals mainly from Europe and Australia. In each study the vast majority of subjects were male (84\%-91\%) and predominately white (81\%-87\%). Baseline characteristics were similar across treatment groups in both studies. Median baseline CD4+ cell counts ranged from 150 to $182 \mathrm{cells} / \mathrm{mm}^{3}$, median baseline viral loads from 4.84 to $4.89 \log 10$ copies/mL, enfuvirtide (T-20, Fuzeon) use from $37 \%$ to $46 \%$ of subjects, and the majority of subjects had 2 or fewer active agents in their OBT $(66 \%-76 \%$ and $62 \%-66 \%$ in MOTIVATE 1 and 2, respectively).

Maraviroc arms in both studies demonstrated highly statistically significant virologic and immunologic benefits compared with the placebo groups. Maraviroc was very well tolerated, with no differences observed in the rate of study discontinuation or grade 3/4 adverse events across treatment arms. Importantly, no excess of cancer diagnoses or liver function abnormalities were observed in the maraviroc groups compared to placebo.

Combined analysis of the two studies was performed to assess maraviroc's contribution to viral suppression. As

Table I 24-week results of phase III maraviroc studies in CCR5-tropic subjects (Lalezari et al 2007; Nelson et al 2007)

\begin{tabular}{|c|c|c|c|}
\hline \multirow[t]{2}{*}{24 week outcome } & \multicolumn{3}{|c|}{ MOTIVATE I and MOTIVATE 2 Results respectively } \\
\hline & Placebo + OBT & MVC once daily + OBT & MVC twice daily + OBT \\
\hline $\begin{array}{l}\text { Mean change in HIV-I viral load from } \\
\text { baseline (log I } 0 \text { copies } / \mathrm{mL})\end{array}$ & $-1.03,-0.93$ & $-1.82,-1.95$ & $-1.95,-1.97$ \\
\hline HIV-I viral load < 400 copies/mL (\%) & $31.4,23.1$ & $54.7^{*}, 55.5^{*}$ & $60.4^{*}, 61.3^{*}$ \\
\hline HIV-I viral load < 50 copies/mL (\%) & $24.6,20.9$ & $42.2^{*}, 40.8 *$ & $48.5,45.6^{*}$ \\
\hline $\begin{array}{l}\text { Mean change in CD4+ cell count } \\
\text { from baseline }\left(\text { cells } / \mathrm{mm}^{3}\right)\end{array}$ & $+52,+64$ & $+107^{*},+112^{*}$ & $+111 *,+102 *$ \\
\hline
\end{tabular}

$*_{\mathrm{p}}<0.001$ in comparison with placebo.

Abbreviations: MVC, maraviroc; OBT, optimized background therapy. 
expected, with an increasing number of active agents in the OBT, the greater the likelihood of reaching an undetectable viral load. Subjects in the maraviroc arms achieved a much higher proportion of complete viral suppression when two or fewer active agents were present in the OBT in comparison with the placebo arm. This is consistent with numerous prior studies demonstrating that two active agents are keys to a successful salvage regimen.

More recent combined 24-week analyses demonstrated results similar to the individual studies (see Table 2; Gulick et al 2007d). While the studies were not designed for a formal comparison of once daily and twice daily maraviroc dosing, higher rates of virologic suppression were observed with twice-daily maraviroc than with once-daily maraviroc in several important subgroups: subjects with viral loads $\geq 100,000$ copies/mL at screening, and also subjects with baseline CD 4 counts $<50$ cells $/ \mathrm{mm}^{3}$. With such robust data, the makers of maraviroc requested and received accelerated approval by the FDA for treatment-experienced patients with inadequate control of multidrug resistant, CCR5-tropic HIV-1. In conjunction with maraviroc's approval, the singlecycle recombinant virus tropism assay (Trofile ${ }^{\circledR}$, Monogram Biosciences South San Francisco, CA, USA) also began its commercial availability (Coakley et al 2005).

While the approval of maraviroc is for the treatment of patients with CCR5-tropic virus, its potential in patients with D/M-tropic virus has also been evaluated. Pfizer study A4001029 is a double-blind placebo controlled study in subjects with $\mathrm{D} / \mathrm{M}$-tropic virus who were randomized to OBT including at least one sensitive drug, plus either maraviroc once daily, maraviroc twice daily, or placebo (Goodrich et al 2007). 186 subjects with $\mathrm{D} / \mathrm{M}$ virus and advanced immunodeficiency, approximate median CD4 cell count of 42 cells $/ \mathrm{mm}^{3}$ and HIV viral load of $5.1 \log$ copies $/ \mathrm{mL}$ within each cohort, were enrolled across the study.

After 48 weeks, the mean viral load decreases were 0.62 $\log$ copies $/ \mathrm{mL}$ in the once-daily maraviroc group, $1.11 \mathrm{log}$
copies/mL in the twice-daily group, and $0.84 \log$ copies $/ \mathrm{mL}$ in the placebo group. These differences were not statistically significant and there was also no substantial difference across study cohorts in the proportion of subjects who achieved an undetectable viral load. However, despite a lack of antiretroviral benefit, CD4 cell counts did rise in both maraviroc cohorts, by 65 cells $/ \mathrm{mm}^{3}$ in the once daily arm and 78 cells $/ \mathrm{mm}^{3}$ in the twice daily arm, whereas the placebo cohort CD4 cell count increase was only 51 cells $/ \mathrm{mm}^{3}$. This finding has generated interest in the possibility that maraviroc, or CCR5 antagonists in general, may have an effect on elevating CD4 cell counts independent of antiviral activity.

Similar rates of adverse effects and deaths were observed, while no cases of lymphoma or adenocarcinoma occurred. Thus, in addition to further reassurance of maraviroc's safety profile, the lack of HIV progression in the D/M group was quite significant and deserves additional investigation as concerns linger regarding the impact of X4-tropic virus.

\section{Consequences of CCR5 antagonist treatment}

A number of concerns, some unique and others general, are germane to the potential use of CCR5 antagonists in patients. As previously discussed, targeting CCR5 leaves a patient without the role of the CCR5 receptor in innate immunity. However, the natural example of the $\triangle 32$ CCR 5 allele and the redundancy of chemokine receptor function provide significant reassurance that blockade should be tolerable. Whether untoward consequences of the iatrogenic loss of the CCR5 receptor's function, including an undue number of severe West Nile virus infections in WNV endemic areas, is a central question that will prove important to CCR5 antagonist use.

Also, just as with all antiretrovirals to date, the issue of viral escape has attended the development of CCR5 antagonists. This concern takes on more substance with CCR5 antagonists than other agents as failure of HAART using a CCR5 antagonist may result in co-receptor switching to

Table 2 Contribution of maraviroc at 24 weeks to viral suppression based on overall susceptibility score of phase III maraviroc studies (Gulick et al 2007d)

\begin{tabular}{|c|c|c|c|}
\hline \multirow{2}{*}{$\begin{array}{l}\text { Number of active agents } \\
\text { in OBT based on overall } \\
\text { susceptibility score }\end{array}$} & \multicolumn{3}{|c|}{ HIV-I viral load $<50$ copies/mL (\%) } \\
\hline & Placebo + OBT & MVC once daily + OBT & MVC twice daily + OBT \\
\hline$\overline{0}$ & 3 & 18 & 29 \\
\hline I & 9 & 43 & 43 \\
\hline 2 & 19 & 52 & 53 \\
\hline$\geq 3$ & 55 & 61 & 58 \\
\hline
\end{tabular}

Abbreviations: MVC, maraviroc; OBT, optimized background therapy. 
CXCR4. Whether de novo or through promotion of minor $\mathrm{D} / \mathrm{M}$ or CXCR4 using variants already present within the patient, co-receptor switching would be a troubling outcome as $\mathrm{D} / \mathrm{M}$ and $\mathrm{CXCR} 4$ tropic viruses have historically, but not uniformly, been associated with accelerated CD4 cell loss and progression to AIDS (Philpott 2003; Daar et al 2007; Goetz et al 2007). Nevertheless it remains possible that emergence of CXCR4 using virus is a consequence and not a cause of severe immunodeficiency.

Some data addressing co-receptor switching in the setting of CCR5 antagonist use have been reported. Analysis of co-receptor switching during early studies of maraviroc suggests that emergent $\mathrm{D} / \mathrm{M}$ and $\mathrm{CXCR} 4$ tropic viruses result from a pre-treatment reservoir not detected by screening tropism assays (Westby et al 2006). During MOTIVATE 1 and 2, maraviroc's phase III trials, 63 of 751 MVC treated subjects failed with $\mathrm{D} / \mathrm{M}$ or X4-tropic virus compared to 35 R5-tropic subjects failing with R5-tropic virus. Reassuringly, patients failing a maraviroc regimen with $\mathrm{D} / \mathrm{M}$ or X4-tropic virus had higher mean increases in CD4 count vs placebo at time of failure (range +37 to +56 cells $/ \mu$ vs +14 cells $/ \mu l$ respectively). Approximately two thirds of these subjects had their D/M or X4 virus return to $\mathrm{R} 5$ tropism prior to going off study (median = 203 days), and there was no association between CXCR4-using virus and AIDS-defining events. Time to failure with a $\mathrm{D} / \mathrm{M}$ or X4-tropic virus was also shorter than for failure with a CCR5-tropic virus. Overall, these results are consistent with the selective and reversible suppression of R5 virus during maraviroc therapy (van der Ryst et al 2007).

In vitro studies have also demonstrated that viral escape may be a consequence of viral adaptation to the conformational change in CCR 5 caused by CCR 5 antagonist binding. Laboratory isolates of HIV-1 have demonstrated the ability to generate mutations in the V3 region of gp120 that allow cell entry via vicriviroc- or maraviroc-bound CCR5 (Pugach et al 2007; Westby et al 2007). Emerging data demonstrate multiple pathways and significant complexity to CCR5 resistance. Preliminarily it appears these mutations are not as stereotypical as those for other antiretroviral agents but rather specific to the env background of the individual virus (Mori et al 2007; Tsibris et al 2007).

\section{Role in HIV-I therapy and conclusions}

Evidenced by the FDA and European Medicines Agency's (EMEA) recent approval of maraviroc (as Selzentry ${ }^{\circledR}$ in the USA and Celsentri ${ }^{\circledR}$ in the EU), CCR5 antagonists are a welcome addition to the care of treatment-experienced patients with CCR5-tropic virus. Their potential role in treatment-naïve patients is unsupported at this time, considering the shortfalls of both maraviroc and vicriviroc based regimens against an EFV based regimen. This is unfortunate, as it may be better to use CCR5 antagonists early in the course of HIV-1 infection treatment when CCR5-tropic virus predominates. Whether patients with $\mathrm{D} / \mathrm{M}$ virus may derive immunologic benefit from a CCR5 antagonist without significant consequence also remains to be seen in large studies.

Additional questions that remain to be answered that will help guide the use of CCR5 antagonists include whether the agents are cross-resistant, synergistic with each other or other agents, and, should CXCR4 antagonists be developed, whether combination X4/R5 inhibitor therapy would have merit. Clearly the addition of another distinct antiretroviral class should be met with celebration, particularly since it is only 10 years after target identification. However further experience and study are needed to clearly define the risks, benefits, and roles that CCR5 antagonists will have in the care of HIV-infected patients.

\section{References}

Anderson J, Akkina R. 2007. Complete knockdown of CCR5 by lentiviral vector-expressed siRNAs and protection of transgenic macrophages against HIV-1 infection. Gene Ther, 14:1287-97.

Baba M, Nishimura O, Kanzaki N, et al. 1999. A small-molecule, nonpeptide CCR5 antagonist with highly potent and selective anti-HIV-1 activity. Proc Natl Acad Sci USA, 96:5698-703.

Baba M, Takashima K, Miyake H, et al. 2005. TAK-652 inhibits CCR5mediated human immunodeficiency virus type 1 infection in vitro and has favorable pharmacokinetics in humans. Antimicrob Agents Chemother, 49:4584-91.

Bartlett JA, DeMasi R, Quinn J, et al. Overview of the effectiveness of triple combination therapy in antiretroviral-naive HIV-1-1-1 infected adults. 2001. AIDS, 15:1369-77.

Blanpain C, Migeotte I, Lee B, et al. 1999. CCR5 binds multiple CC-chemokines: MCP-3 acts as a natural antagonist. Blood, 94:1899-905.

Brumme ZL, Goodrich J, Mayer HB, et al. 2005. Molecular and clinical epidemiology of CXCR4-using HIV-1 in a large population of antiretroviral-naïve individuals. J Infect Dis, 192:466-74.

Chan DC, Fass D, Berger JM, et al. 1997. Core structure of gp41 from the HIV envelope glycoprotein. Cell, 89:263-73.

Chan DC, Kim PS. 1998. HIV entry and its inhibition. Cell, 93:681-4.

Coakley E, Petropoulos CJ, Whitcomb JM. 2005. Assessing chemokine co-receptor usage in HIV. Curr Opin Infect Dis, 18:9-15.

Cocchi F, DeVico AL, Garzino-Demo A, et al. 1995. Identification of RANTES, MIP-1 alpha, and MIP-1 beta as the major HIV-suppressive factors produced by CD8+ T cells. Science, 270:1811-15.

Connor RI, Sheridan KE, Ceradini D, et al. 1997. Change in coreceptor use correlates with disease progression in HIV-1 infected individuals. $J$ Exp Med, 185:621-8.

Cooper D, Gatell J, Rockstroh J, et al. 2007. Results from BENCHMRK-1, a phase III study evaluating the efficacy and safety of MK-0518, a novel HIV-1 integrase inhibitor, in patients with triple-class resistant virus. Program and abstracts of the 14th Conference on Retroviruses and Opportunistic Infections, Los Angeles, CA, Abstract 105aLB.

Crabb C. 2006. GlaxoSmithKline ends aplaviroc trials. AIDS, 20:641. 
Daar ES, Kesler KL, Petropoulos CJ, et al. 2007. Hemophilia Growth and Development Study. Baseline HIV type 1 coreceptor tropism predicts disease progression. Clin Infect Dis, 45:643-9.

Dalgleish AG, Beverley PC, Clapham PR, et al. 1984. The CD4 (T4) antigen is an essential component of the receptor for the AIDS retrovirus. Nature, 312:763-7.

Dean M, Carrington M, Winkler C, et al. 1996. Genetic restriction of HIV-1 infection and progression to AIDS by a deletion allele of the CKR5 structural gene: Hemophilia Growth and Development Study, Multicenter AIDS Cohort Study, Multicenter Hemophilia Cohort Study, San Francisco City Cohort, ALIVE Study. Science, 273:1856-62.

Deeks SG. 2006. Challenges of developing R5 inhibitors in antiretroviral naive HIV-infected patients. Lancet, 367:711-3.

Dorr P, Westby M, Dobbs S, et al. 2005. Maraviroc (UK-427,857), a potent, orally bioavailable, and selective small-molecule inhibitor of chemokine receptor CCR5 with broad-spectrum anti-human immunodeficiency virus type 1 activity. Antimicrob Agents Chemother, 49:4721-32.

Dragic T, Litwin V, Allaway GP, et al. 1996. HIV-1 entry into CD4+ cells is mediated by the chemokine receptor CC-CKR-5. Nature, 381:667-73.

Dragic T, Trkola A, Thompson DA, et al. 2000. A binding pocket for a small molecule inhibitor of HIV-1 entry within the transmembrane helices of CCR5. Proc Natl Acad Sci USA, 97:5639-44.

Eckert DM, Kim PS. 2001. Mechanisms of viral membrane fusion and its inhibition. Annu Rev Biochem, 70:777-810.

Fätkenheuer G, Pozniak AL, Johnson MA, et al. 2005. Efficacy of shortterm monotherapy with maraviroc, a new CCR5 antagonist, in patients infected with HIV-1. Nat Med, 11:1170-2.

Fischereder M, Luckow B, Hocher B, et al. 2001. CC chemokine receptor 5 and renal-tranplant survival. Lancet, 357:1758-61.

Galvani AP, Slatkin M. 2003. Evaluating plague and smallpox as historical selective pressures for the CCR5- 32 HIV-resistance allele. Proc Natl Acad Sci USA, 100:15276-9.

Glass WG, McDermott DH, Lim JK, et al. 2006. CCR5 deficiency increases risk of symptomatic West Nile virus infection. J Exp Med, 203:35-40.

Goetz MB, Leduc R, Kostman JR, et al. 2007. Prediction of disease progression by HIV coreceptor tropism in persons with untreated chronic HIV infection. Program and abstracts of the 47th Interscience Conference on Antimicrobial Agents and Chemotherapy, Chicago, Ill. Abstract H-1027.

Goodrich JM, Saag M, van der Ryst E, et al. 2007. 48-week safety and efficacy of maraviroc, a novel ccr5 antagonist, in combination with optimized background therapy (OBT) for the treatment of antiretroviralexperienced patients infected with dual/mixed-tropic HIV-1. Program and abstracts of the 45th Annual Infectious Diseases Society of America, San Diego, CA, Abstract LB-2.

Greaves W, Landovitz R, Fätkenheuer G, et al. 2006. Late Virologic breakthrough in treatment-naive patients on a regimen of combivir + vicriviroc. Program and abstracts of the 13th Conference on Retroviruses and Opportunistic Infections, Denver, CO, Abstract 161LB.

Gulick R, Su Z, Flexner C, et al. 2007a. Phase II study of the safety and efficacy of vicriviroc in HIV-infected treatment-experienced subjects: ACTG 5211. J Infect Dis, 196:304-12.

Gulick R, Su Z, Flexner C, et al. for the ACTG 5211 Team. 2007b. ACTG 5211: phase II study of the safety and efficacy of vicriviroc (VCV) in HIV-infected treatment-experienced subjects: 48 week results. Program and abstracts of the 4th International AIDS Society Conference on HIV Pathogenesis, Treatment and Prevention, Sydney, Australia, Abstract TUAB102.

Gulick R, Haas D, Collier A, et al. 2007c. Two-year follow-up of treatmentexperienced patients on vicriviroc (VCV). Program and abstracts of the 47th Interscience Conference on Antimicrobial Agents and Chemotherapy, Chicago, IL, Abstract H-1030.

Gulick RM, van der Ryst E, Lampiris H, et al. 2007d. Efficacy and safety of oncedaily (QD) compared with twice-daily (BID) maraviroc plus optimized background therapy (OBT) in treatment-experienced patients infected with CCR5-tropic-HIV-1: 24-week combined analysis of the MOTIVATE 1 and 2 studies. Program and abstracts of the 4th IAS Conference on HIV Pathogenesis, Treatment and Prevention. Abstract WEPEB116LB.
Guo Q, Ho HT, Dicker I, et al. 2003. Biochemical and genetic characterizations of a novel human immunodeficiency virus type 1 inhibitor that blocks gp120-CD4 interactions. J Virol, 77:10528-36.

Hammer SM, Saag MS, Schechter M, et al. 2006. International AIDS SocietyUSA panel. Treatment for adult HIV-1 infection: 2006 recommendations of the International AIDS Society-USA panel. JAMA, 296:827-43.

Hartley O, Gaertner H, Wilken J, et al. 2004. Medicinal chemistry applied to a synthetic protein: development of highly potent HIV entry inhibitors. Proc Natl Acad Sci USA, 101:16460-5.

Holmberg SD, Hamburger EM, Moorman AC, et al. 2003. Factors associated with maintenance of long-term plasma human immunodeficiency virus RNA suppression. Clin Infect Dis, 37:702-7.

Huang Y, Paxton WA, Wolinsky SM, et al. 1996. The role of a mutant CCR5 allele in HIV-1 transmission and disease progression. Nat Med, $2: 1240-3$.

Jacobson JM, Israel RJ, Lowy I, et al. 2004. Treatment of advanced human immunodeficiency virus type 1 disease with the viral entry inhibitor PRO 542. Antimicrob Agents Chemother, 48:423-9.

Jacobson JM, Thompson M, Saag MS, et al. 2007. Antiretroviral Activity and Pharmacodynamics of PRO 140, a CCR5 Monoclonal Antibody, in HIV-Infected Individuals. Program and abstracts of the 47th Annual Interscience Conference on Antimicrobial Agents and Chemotherapy, Chicago, IL, Abstract H-716.

Ji C, Zhang J, Dioszegi M, et al. 2007. CCR5 small-molecule antagonists and monoclonal antibodies exert potent synergistic antiviral effects by cobinding to the receptor. Mol Pharmacol, 72:18-28.

Jouvenot Y, Perez E, Urnov FD, et al. 2005. Towards Gene Knock Out Therapy for AIDS/HIV: Targeted Disruption of CCR5 Using Engineered Zinc Finger Protein Nucleases (ZFNs). Program and abstracts of the 45th Annual Interscience Conference on Antimicrobial Agents and Chemotherapy, Washington, DC, Abstract H-1084.

Kadow J, Wang HG, Lin PF. 2006. Small-molecule HIV-1 gp120 inhibitors to prevent HIV-1 entry: an emerging opportunity for drug development. Curr Opin Investig Drugs, 7:721-6.

Koot M, Keet IP, Vos AH, et al. 1993. Prognostic value of HIV-1 syncytiuminducing phenotype for rate of CD4+ cell depletion and progression to AIDS. Ann Intern Med, 118:681-8.

Kuhmann SE, Pugach P, Kunstman KJ, et al. 2004. Genetic and phenotypic analyses of human immunodeficiency virus type 1 escape from a small-molecule CCR5 inhibitor. $J$ Virol, 78:2790-807. Erratum in: J Virol, 2005. 78:6706.

Kwong PD, Wyatt R, Robinson J, et al. 1998. Structure of an HIV gp120 envelope glycoprotein in complex with the CD4 receptor and a neutralizing human antibody. Nature, 393:648-59.

Lalezari J, Thompson M, Kumar P, et al. 2005. Antiviral activity and safety of 873140 , a novel CCR5 antagonist, during short-term monotherapy in HIV-infected adults. AIDS, 19:1443-8.

Lalezari J, Lederman M, Yadavalli G, et al. 2006. A phase 1, dose-escalation, placebo-controlled study of a fully human monoclonal antibody (CCR5mAb004) against CCR5 in patients with CCR5-tropic HIV-1 infection. Program and abstracts of the 46th Interscience Conference on Antimicrobial Agents and Chemotherapy, San Francisco, CA, Abstract H-1668.

Lalezari J, Goodrich J, DeJesus E, et al. 2007. MOTIVATE 1. USA and Canada. Efficacy and safety of maraviroc plus optimized background therapy in viremic, ART-experienced patients infected with CCR5tropic HIV-1: 24-week results of phase 2b/3 studies. Program and abstracts of the 14th Conference on Retroviruses and Opportunistic Infections, Los Angeles, CA, Abstract 104bLB.

Lapidot T. 2001. Mechanism of human stem cell migration and repopulation of NOD/SCID and B2mnull NOD/SCID mice. The role of SDF1/CXCR4 interactions. Ann NY Acad Sci, 938:83-95.

Liu R, Paxton WA, Choe S, et al. 1996. Homozygous defect in HIV-1 coreceptor accounts for resistance of some multiply-exposed individuals to HIV-1 infection. Cell, 86:367-77.

Luther SA, Cyster JG. 2001. Chemokines as regulators of T cell differentiation. Nat Immunol, 2:102-7. 
Maeda K, Yoshimura K, Shibayama S, et al. 2001. Novel low molecular weight spirodiketopiperazine derivatives potently inhibit R5 HIV-1 infection through their antagonistic effects on CCR5. J Biol Chem, 276:35194-200

Maeda K, Nakata H, Koh Y, et al. 2004. Spirodiketopiperazine-based CCR5 inhibitor which preserves CC-chemokine/CCR5 interactions and exerts potent activity against R5 human immunodeficiency virus type 1 in vitro. J Virol, 78:8654-62.

Mani M, Kandavelou K, Dy FJ, et al. 2005. Design, engineering, and characterization of zinc finger nucleases. Biochem Biophys Res Commun, 335:447-57.

Markowitz M, Mohri H, Mehandru S, et al. 2005. Infection with multidrug resistant, dual-tropic HIV-1 and rapid progression to AIDS: a case report. Lancet, 365:1031-8.

Marozsan AJ, Kuhmann SE, Morgan T, et al. 2005. Generation and properties of a human immunodeficiency virus type 1 isolate resistant to the small molecule CCR5 inhibitor, SCH-417690 (SCH-D). Virology, 338:182-99.

Melby T, Despirito M, Demasi R, et al. 2006. HIV-1 coreceptor use in tripleclass treatment-experienced patients: baseline prevalence, correlates, and relationship to enfuvirtide response. J Infect Dis, 194:238-46.

Mocroft A, Phillips AN, Friis-Moller N, et al. 2002. Response to antiretroviral therapy among patients exposed to three classes of antiretrovirals: results from the EuroSIDA Study. Antivir Ther, 1:21-30.

Mori J, Mosley M, Lewis M, et al. 2007. Characterization of maraviroc resistance in patients failing treatment with CCR5-tropic virus in MOTIVATE 1 and MOTIVATE 2. Program and abstracts of the 16th International HIV Drug Resistance Workshop, Barbados, West Indies. Abstract 10.

Mosley M, Smith-Burchnell C, Mori J, et al. 2006. Resistance to the CCR5 antagonist maraviroc is characterized by dose-response curves that display a reduction in maximal inhibition. Program and abstracts of the 13th Conference on Retroviruses and Opportunistic Infections, Denver, Colorado. Abstract 598.

Moyle GJ, Wildfire A, Mandalia S, et al. 2005. Epidemiology and predictive factors for chemokine receptor use in HIV-1 infection. $J$ Infect Dis, 191:866-72.

Moyle GJ, DeJesus E, Boffi to M, et al. 2007. CXCR4 antagonism: proof of activity with AMD11070. Program and abstracts of the 14th Conference on Retroviruses and Opportunistic Infections, Los Angeles, CA, Abstract 511

Murga JD, Franti M, Pevear DC, et al. 2006. Potent antiviral synergy between monoclonal antibody and small-molecule CCR5 inhibitors of human immunodeficiency virus type 1 . Antimicrob Agents Chemother, 50:3289-96.

Nakata H, Maeda K, Miyakawa T, et al. 2005. Potent anti-R5 human immunodeficiency virus type 1 effects of a CCR5 antagonist, AK602/ ONO4128/GW873140, in a novel human peripheral blood mononuclear cell nonobese diabetic-SCID, interleukin-2 receptor gammachain-knocked-out AIDS mouse model. J Virol, 79:2087-96.

Nelson M, Fatkenheuer G, Konourina I, et al. 2007. MOTIVATE 2. Europe, Australia and USA. Efficacy and safety of maraviroc plus optimized background therapy in viremic, ART-experienced patients infected with CCR5-tropic HIV-1: 24-week results of phase 2b/3 studies. Program and abstracts of the 14th Conference on Retroviruses and Opportunistic Infections, Los Angeles, CA, Abstract 104aLB.

Oldfield V, Keating GM, Plosker G. 2005. Enfuvirtide: a review of its use in the management of HIV infection. Drugs, 65:1139-60.

Olson WC, Rabut GE, Nagashima KA, et al. 1999. Differential inhibition of human immunodeficiency virus type 1 fusion, gp120 binding, and CC-chemokine activity by monoclonal antibodies to CCR5. J Virol, 735:4145-55

Oppermann M. 2004. Chemokine receptor CCR5: insights into structure, function, and regulation. Cell Signal, 16:1201-10.

Palella FJ Jr, Delaney KM, Moorman AC, et al. 1998. Declining morbidity and mortality among patients with advanced human immunodeficiency virus infection. HIV-1 Outpatient Study Investigators. N Engl J Med, 338:853-60
Pastore C, Nedellec R, Ramos A, et al. 2006. Human immunodeficiency virus type 1 coreceptor switching: V1/V2 gain-of-fitness mutations compensate for V3 loss-of-fitness mutations. $J$ Virol, 80:750-8.

Philpott SM. 2003. HIV-1 coreceptor usage, transmission, and disease progression. Curr HIV Res, 1:217-27.

Prahalad S. 2006. Negative association between the chemokine receptor CCR5-[Delta]32 polymorphism and rheumatoid arthritis: a metaanalysis. Genes Immun, 7:264-8.

Pugach P, Marozsan AJ, Ketas TJ, et al. 2007. HIV-1 clones resistant to a small molecule CCR5 inhibitor use the inhibitor-bound form of CCR5 for entry. Virology, 361:212-28.

Qin XF, An DS, Chen IS, et al. 2003. Inhibiting HIV-1 infection in human T cells by lentiviral-mediated delivery of small interfering RNA against CCR5. Proc Natl Acad Sci USA, 100:183-8.

Reynes J, Rouzier R, Kanouni T, et al. 2002. SCH C: Safety and antiviral effects of a CCR5 receptor antagonist in HIV-1-infected subjects. Program and abstracts of the 9th Conference on Retroviruses and Opportunistic Infections. Seattle, WA, Abstract 1.

Roschke V, Clark S, Branco L, et al. 2004. Characterization of a panel of novel human monoclonal antibodies that specifically antagonize CCR5 and Block HIV-1 entry. Program and abstracts of the 44th Interscience Conference on Antimicrobial Agents and Chemotherapy, Washington, D.C., Abstract \#2871.

Ruffing N, Sullivan N, Sharmeen L, et al. 1998. CCR5 has an expanded ligand-binding repertoire and is the primary receptor used by MCP-2 on activated T cells. Cell Immunol, 189:160-8.

Saag M, Ive P, Heera J, et al. 2007. A multicenter, randomized, double-blind, comparative trial of a novel CCR5 antagonist, maraviroc versus efavirenz, both in combination with Combivir (zidovudine [ZDV]/lamivudine [3TC]), for the treatment of antiretroviral naive patients infected with R5 HIV 1: Week 48 results of the MERIT study. Program and abstracts of the 4th IAS Conference on HIV Pathogenesis, Treatment and Prevention, Sydney, Australia, Abstract WESS104.

Sabeti PC, Walsh E, Schaffner SF, et al. 2005. The case for selection at CCR5-Delta32. PLoS Biol, 3:e378.

Samson M, Libert F, Doranz BJ, et al. 1996. Resistance to HIV-1 infection in Caucasian individuals bearing mutant alleles of the CCR- 5 chemokine receptor gene. Nature, 382:722-5.

Schooley RT, Mellors JW. 2007. No cure yet for HIV-1-1, but therapeutic research presses on. J Infect Dis, 195:770-2.

Schurmann D, Fätkenheuer G, Reynes J, et al. 2007. Antiviral activity, pharmacokinetics and safety of vicriviroc, an oral CCR5 antagonist, during 14-day monotherapy in HIV-infected adults. AIDS, 21:1293-9.

Shet A, Berry L, Mohri H, et al. 2006. Tracking the prevalence of transmitted antiretroviral drug-resistant HIV-1-1: a decade of experience. J Acquir Immune Defic Syndr, 41:439-46.

Signoret N, Hewlett L, Wavre S, et al. 2005. Agonist-induced endocytosis of CC chemokine receptor 5 is clathrin dependent. Mol Biol Cell, 16:902-17.

Simmons G, Clapham PR, Picard L, et al. 1997. Potent inhibition of HIV-1 infectivity in macrophages and lymphocytes by a novel CCR5 antagonist. Science, 276:276-9.

Starr-Spires LD, Collman RG. 2002. HIV-1 entry and entry inhibitors as therapeutic agents. Clin Lab Med, 22:681-701.

Steigbigel R, Kumar P, Eron J, et al. 2007. Results from BENCHMRK-2, a phase III study evaluating the efficacy and safety of MK-0518, a novel HIV-1 integrase inhibitor, in patients with triple-class resistant virus. Program and abstracts of the 14th Conference on Retroviruses and Opportunistic Infections, Los Angeles, CA, Abstract 105bLB.

Stephens JC, Reich DE, Goldstein DB, et al. 1998. Dating the origin of the CCR5-Delta32 AIDS-resistance allele by the coalescence of haplotypes. Am J Hum Genet, 62:1507-15.

Strizki JM, Xu S, Wagner NE, et al. 2001. SCH-C (SCH 351125), an orally bioavailable, small molecule antagonist of the chemokine receptor CCR5, is a potent inhibitor of HIV-1 infection in vitro and in vivo. Proc Natl Acad Sci USA, 98:12718-23. 
Strizki JM, Tremblay C, Xu S, et al. 2005. Discovery and characterization of vicriviroc ( $\mathrm{SCH} 417690$ ), a CCR5 antagonist with potent activity against human immunodeficiency virus type 1. Antimicrob Agents Chemother, 49:4911-9.

Takashima K, Miyake H, Kanzaki N, et al. 2005. Highly potent inhibition of human immunodeficiency virus type 1 replication by TAK-220, an orally bioavailable small-molecule CCR5 antagonist. Antimicrob Agents Chemother, 49:3474-82.

Thio CL, Astemborski J, Bashirova A, et al. 2007. Genetic protection against hepatitis B virus conferred by CCR5Delta32: Evidence that CCR5 contributes to viral persistence. J Virol, 81:441-5.

Tsamis F, Gavrilov S, Kajumo F, et al. 2003. Analysis of the mechanism by which the small-molecule CCR5 antagonists SCH-351125 and SCH-350581 inhibit human immunodeficiency virus type 1 entry. $J$ Virol, 77:5201-8.

Tsibris AMN, Gulick RM, Su Z, et al. 2007. In vivo emergence of HIV-1 resistance to the CCR5 antagonist vicriviroc: findings from ACTG A5211. Program and abstracts of the 16th International HIV Drug Resistance Workshop, Barbados, West Indies, Abstract 13.

Unutmaz D, KewalRamani VN, Littman DR. 1998. G protein-coupled receptors in HIV and SIV entry: new perspectives on lentivirus-host interactions an on the utility of animal models. Semin Immunol, 10:225-36.

van der Ryst E, Westby M. 2007. Changes in HIV-1 co-receptor tropism for patients participating in the maraviroc Motivate 1 and 2 clinical trials. Program and abstracts of the 47th Interscience Conference on Antimicrobial Agents and Chemotherapy, Chicago, IL, Abstract H-715.
Walensky RP, Paltiel AD, Losina E, et al. 2006. The survival benefits of AIDS treatment in the United States. J Infect Dis, 194:11-9.

Westby M, Lewis M, Whitcomb J, et al. 2006. Emergence of CXCR4-using human immunodeficiency virus type 1 (HIV-1) variants in a minority of HIV-1-infected patients following treatment with the CCR5 antagonist maraviroc is from a pretreatment CXCR4-using virus reservoir. $J$ Virol, 80:4909-20.

Westby M, Smith-Burchnell C, Mori J, et al. 2007. Reduced maximal inhibition in phenotypic susceptibility assays indicates that viral strains resistant to the CCR5 antagonist maraviroc utilize inhibitor-bound receptor for entry. $J$ Virol, 81:2359-71.

Wilkin TJ, Su Z, Kuritzkes DR, et al. 2007. HIV type 1 chemokine coreceptor use among antiretroviral-experienced patients screened for a clinical trial of a CCR5 inhibitor: AIDS Clinical Trial Group A5211. $J$ Infect Dis, 44:591-5.

Wong MM, Fish EN. 2003. Chemokines: attractive mediators of the immune response. Semin Immunol, 15:5-14.

Wood A, Armour D. 2005. The discovery of the CCR5 receptor antagonist, UK-427,857, a new agent for the treatment of HIV infection and AIDS. Prog Med Chem, 43:239-71.

Zhao J, Ma L, Wu YL, et al. 1998. Chemokine receptor CCR5 functionally couples to inhibitory $\mathrm{G}$ proteins and undergoes desensitization. $J$ Cell Biochem, 71:36-45. 
